\section{Hacia un marco conceptual para repensar la accesibilidad cultural}

\author{
Towards a theoretical framework for rethinking \\ cultural accessibility
}

\author{
Por um marco conceitual para repensar a \\ acessibilidade cultural
}

Fernando Landini 1,2

Valeria González Cowes 3

Eliana D'Amore 1,2

\footnotetext{
${ }^{1}$ Consejo Nacional de Investigaciones Científicas y Técnicas, Buenos Aires, Argentina.

2 Universidad de la Cuenca del Plata, Corrientes, Argentina.

${ }^{3}$ Facultad de Psicología, Universidad de Buenos Aires, Buenos Aires, Argentina.

Correspondencia

F. Landini

Consejo Nacional de

Investigaciones Científicas y

Técnicas.

Terrero 1165, 3o A, Buenos

Aires / N/A - 1416, Argentina.

landini_fer@hotmail.com
}

\begin{abstract}
Health services accessibility is a key health policy issue. However, few in-depth studies have addressed it theoretically. Most distinguish between availability, accessibility, and acceptability, or between geographic, financial, administrative, and cultural accessibility. We discuss and analyze the concept of accessibility as conflictive articulation between supply and demand in health. The article addresses the importance of cultural accessibility, rethinking it as a social interface, i.e., a social arena with clashing worldviews (namely, those of physicians and patients). The approach sheds light on the complex processes of grasping, translating, and reshaping knowledge and recommendations within such interaction.
\end{abstract}

Health Services Accessibility; Cultural Factors; Psychological Phenomena and Processes; Communication Barriers

\section{Resumen}

La accesibilidad de la población al sistema de salud constituye una de las principales preocupaciones de las políticas sanitarias. No obstante, son pocos los trabajos que profundizan conceptualmente en dicha noción. La mayor parte de ellos diferencia entre disponibilidad, accesibilidad y aceptabilidad, o entre accesibilidad geográfica, financiera, administrativa y cultural. En el presente trabajo se discute y analiza la noción de accesibilidad, definiéndosela como un proceso de articulación conflictiva entre demanda y oferta en salud. A la vez, se focaliza en la noción de accesibilidad cultural, la cual es repensada como una interfaz social, es decir, como un espacio conflictivo en el que se articulan marcos de sentido diferentes, en este caso el de los profesionales y el de los pacientes. Esto permite abordar los procesos complejos de apropiación, traducción y reconfiguración de los conocimientos y recomendaciones que se dan en este vínculo.

Accesibilidad a los Servicios de Salud; Factores Culturales; Fenómenos y Procesos Psicológicos; Barreras de Comunicación 


\section{Introducción}

La accesibilidad de la población al sistema de salud constituye uno de los elementos fundamentales de la noción misma de Atención Primaria de la Salud (APS), como fue definida en la Declaración de Alma-Ata de $1978{ }^{1}$ y reafirmada por la Organización Panamericana de la Salud (OPS) en el 2007 2. Sin embargo, si bien el concepto de accesibilidad ha sido utilizado en diversos trabajos científicos y documentos institucionales, muchas veces se utiliza de manera imprecisa o con limitada profundidad conceptual, particularmente en lo que se refiere a la dimensión cultural de la accesibilidad.

Así, se establece como objetivo de este trabajo analizar y discutir críticamente la noción de accesibilidad, entendida como proceso conflictivo de articulación entre demanda y oferta en salud. En este contexto, se hace énfasis en la dimensión psico-socio-cultural de la accesibilidad, a la que se propone abordar a partir del concepto de interfaz social, en tanto espacio de actualización de discontinuidades sociales, en el que se encuentran actores que poseen diferentes racionalidades culturales o que forman parte de grupos sociales diferentes.

Para esto se realizó una revisión crítica de bibliografía para la cual se utilizaron las bases de revistas científicas Directory of Open Access Journals, EBSCO, REDALYC y SciELO. Las palabras introducidas en los motores de búsqueda fueron "acceso" y "accesibilidad" (y sus traducciones al portugués e inglés), a las que se añadían de manera flexible otras como "salud" y "sistema de salud", con el fin de obtener resultados más específicos. A la vez, durante el análisis se agregó la búsqueda de trabajos sobre "relación médicopaciente", debido a que algunos referidos a esta temática resaltaron por su interés durante la revisión previa. El presente trabajo corresponde a una revisión crítica de bibliografía referida a la accesibilidad al sistema de salud y a temas conexos.

\section{El concepto de accesibilidad}

La accesibilidad de la población al sistema de salud es una de las principales preocupaciones de las políticas sanitarias 3 , constituye un área prioritaria de investigación 4 , a la vez que una variable de gran impacto en la salud de la población 5,6, razón por la cual existen numerosos estudios que se han dedicado a abordar esta temática. No obstante, una parte importante de ellos tienden a utilizar la noción sin definirla o fundarla a nivel teórico con solidez 7,8,9,10,11. Solitario et al. 12 (p.
98) argumentan que el concepto de accesibilidad “aparece en los textos dándolo por sentado, como si existiera un acuerdo básico entre actores o se encontrara naturalizado". En la misma línea Comes \& Stolkiner 13 también señalan que, muchas veces, los trabajos que abordan este tema sólo presentan definiciones simplificadas sin referencias bibliográficas que las sostengan.

La accesibilidad ha sido definida de diferentes maneras 7,8,9,10,11,12,13,14. A la vez, posee límites borrosos con otros conceptos relacionados, como los de acceso, uso o calidad de los servicios de salud, los cuales algunos autores utilizan como sinónimos 3,5. Como señalan Travassos \& Martins 14, existen dos líneas conceptuales para definir la accesibilidad. Por un lado, están quienes consideran la accesibilidad como una característica de la oferta de los servicios de salud, entendiéndola como aquellas características de los servicios que facilitan u obstaculizan el hecho de que los usuarios puedan acceder y recibir la atención que necesitan 15 . No obstante, la mayor parte de los autores tiende a pensar la accesibilidad en términos del ajuste entre las características de los servicios y sus profesionales, y las necesidades, situaciones o particularidades de la población que se busca beneficiar 5,16,17,18,19. En esta línea, se tiende a concebir la accesibilidad como la posibilidad y/o capacidad de los usuarios de recibir cuidados de salud de manera ágil cuando resulta necesario 20,21 , lo que permite centrarse en la articulación entre la oferta de los servicios de salud y las características de la demanda.

Vista así, la noción de accesibilidad se muestra como un concepto relacional, ya que se refiere al vínculo que se establece entre los sujetos y los servicios de salud, siendo imposible plantearse el acceso sin considerar la articulación entre ambos 22,23. De este modo, cobra sentido plantearse el concepto de barreras de acceso, entendidas como aquellos factores que se colocan entre los usuarios y los servicios obstaculizando o limitando la posibilidad de lograr la atención necesaria 13,24,25. Así, la accesibilidad quedaría definida como la articulación o ajuste entre las características de la oferta (es decir, del sistema de salud y sus profesionales) y las características y necesidades de la demanda, pudiendo existir barreras que limiten la posibilidad de acceder a una atención satisfactoria en el ámbito de la salud.

\section{¿Acceso al sistema de salud o a sus beneficios?}

En el contexto de una concepción relacional de la accesibilidad, la mayoría de los autores tiende a 
concebirla como la posibilidad de las personas de acceder a alguna de las instancias del sistema de salud 14,16. En cambio, otros se plantean el acceso no al sistema, sino al tratamiento que los usuarios requieren o necesitan 5 . En cualquier caso, el foco queda ubicado en la llegada, ingreso o atención brindada en el sistema de salud. Ahora bien, bajo esta conceptualización, el impacto que esa llegada, ingreso o atención recibida por parte del sistema de salud tiene en términos de la salud de los usuarios, aparece como un elemento externo o ajeno a la noción de accesibilidad. Así, cabe preguntarse por qué el foco debe ser puesto en el sistema de salud y no en las mejoras de salud que los usuarios pueden obtener a partir de su entrada o contacto con el sistema sanitario. De hecho, si bien pocos, hay autores o instituciones que tienden a incluir esta dimensión cuando se refieren al acceso de la población al sector salud. Este es el caso del Instituto de Medicina de los Estados Unidos, el cual propone considerar al acceso como el uso de los servicios de salud en el tiempo adecuado para obtener el mejor resultado posible ${ }^{14}$. Aquí, la obtención del mejor resultado posible estaría incluyendo la dimensión del impacto en la salud como resultado del acceso al sistema.

Se argumenta la existencia de una falacia, como consecuencia de la metáfora espacial, subyacente a la idea de "accesibilidad" más frecuentemente utilizada. La accesibilidad, entendida como concepto relacional, construye la idea de la existencia de dos elementos o actores, los usuarios y el sistema de salud. Así, se conceptualiza intuitivamente como accesibilidad la posibilidad de atravesar ese espacio imaginario (incluidas las barreras) que existen entre uno y otro. Un ejemplo interesante de esto es un estudio que plantea la accesibilidad a servicios comunitarios de salud mental en términos de un sistema de "puertas abiertas" 16, lo que hace referencia al "dejar entrar" a los usuarios sin ponerle trabas. O el trabajo de Girondi \& Santos 26, quienes estudian las barreras arquitectónicas que impiden a pacientes mayores entrar en los servicios. Así, esta intuición "espacial" o incluso "geográfica" de la accesibilidad, centrada en el acceso físico a los servicios de salud, tiende a invisibilizar el impacto que tiene la atención recibida en la salud. En efecto, podría tratarse de una atención con bajos estándares de calidad que genere impactos limitados (o incluso negativos) en la salud de los pacientes. O podría ser que las recomendaciones y tratamientos terapéuticos propuestos por los profesionales no se adecuaran a las posibilidades reales o a la cultura de los beneficiarios, lo que llevaría a bajos porcentajes de adherencia a los tratamientos $25,27,28$. Por otra parte, esta metáfora espacial también lleva a pensar que son los usuarios (quienes tienen la capacidad de desplazarse) los "responsables" de llegar al sistema (estático y expectante, más o menos amigable para acogerlos); lo que favorece que la responsabilidad de actuar sea delegada o depositada imaginariamente en los usuarios, quedando en la oscuridad la capacidad activa del sistema de salud. En consecuencia, dadas las limitaciones de pensar la accesibilidad a partir de la imagen geográfica o espacial que induce el concepto, se propone pensarla como la articulación o relación (muchas veces negociada y conflictiva) entre las características del sistema de salud y sus profesionales, y las características y necesidades de la población, vínculo que se espera que permita una atención médicamente fundada a la vez que cultural y contextualmente apropiada, que genere beneficios en la salud y el bienestar de los pacientes. Acceder al sistema de salud no es suficiente 29. Así, convendría hablar de accesibilidad (o acceso) no ya al sistema de salud, sino a los beneficios que éste puede proveer en términos de salud, dejando de asumir que el acceso al sistema implica, en sí mismo, un impacto positivo en la salud de las personas.

\section{Tipologías de accesibilidad}

Si bien en términos generales la noción de accesibilidad no ha sido desarrollada en profundidad a nivel conceptual 15, los trabajos que abordan el tema sí suelen contener diversas tipologías 16 que abordan de manera práctica el concepto. Dos son las tipologías más mencionadas en la bibliografía. La primera conceptualiza el acceso diferenciando entre disponibilidad, accesibilidad y aceptabilidad de los servicios de salud 30,31,32. Considera "disponibles" a los servicios de salud cuando estos existen en un territorio determinado ${ }^{31}$, lo que requiere tener en cuenta tanto su cantidad como tipo ${ }^{14}$. De igual forma, se los define como 'accesibles', si las personas tienen los medios necesarios para utilizar esos servicios 31 , particularmente, en lo referido a su distribución y ubicación geográfica en relación a los pacientes 14 . Finalmente, son "aceptables" si responden a las necesidades, expectativas y valores de los beneficiarios, invitando a continuar con la atención recibida 31 , lo que se conecta de manera directa, tanto con las actitudes de los beneficiarios como de los profesionales de la salud 14 en el momento del encuentro entre ambos 17 . El segundo abordaje diferencia entre tres, cuatro o cinco dimensiones de la accesibilidad, siendo la alternativa más utilizada la de diferenciar entre accesibilidad geográfica, financiera, adminis- 
trativa y cultural. No obstante, es respecto a la accesibilidad cultural donde aparecen más diferencias. En efecto, hay autores que la excluyen de su análisis, quedándose sólo con las restantes dimensiones 5,33. Por su parte Sales Clementino \& Nunes 18 la conceptualizan como accesibilidad sociocultural, ampliando su alcance. No obstante, es el equipo de Stolkiner el que realiza la contribución más novedosa, al complementar la idea de accesibilidad cultural con lo que denominan accesibilidad simbólica13,22,23.

Siguiendo lo propuesto por diferentes autores 13,22 , se entiende por accesibilidad geográfica a aquella relacionada con las distancias entre los beneficiarios y los servicios, y la posibilidad de cubrir ese recorrido a partir de las vías de comunicación existentes y los transportes disponibles. La accesibilidad económica refiere a la capacidad financiera de las personas en relación al costo del transporte para llegar a los centros de salud, el valor de la atención recibida y el dinero necesario para la compra de medicamentos, realización de estudios y otros costos derivados. La accesibilidad administrativa atiende a las trabas burocráticas relacionadas con el acceso al servicio, como horarios de atención o sistemas de asignación de turnos. Finalmente, la accesibilidad cultural focaliza en los hábitos y costumbres de la población y las diferencias existentes a nivel cultural entre los profesionales y la organización del servicio, por un lado, y los beneficiarios, por el otro.

Resulta de interés clarificar el concepto de accesibilidad simbólica y su relación con el concepto de accesibilidad cultural. A partir de diversos trabajos de investigación llevados adelante por Stolkiner y su equipo, se identificó que el modo en que las personas conceptualizan la atención gratuita en el ámbito de salud público, como derecho o como acto de beneficencia por su condición de pobreza, puede constituirse en una barrera para acceder al sistema de salud y a sus beneficios 22,23. Ellas destacan que los imaginarios sociales y las representaciones de los sujetos también pueden constituirse en barreras específicas que deben ser tomadas en cuenta. Para esto acuñan la idea de accesibilidad simbólica, aludiendo al universo simbólico o subjetivo que organiza la vida de los sujetos. Resulta interesante mencionar que la propuesta de la accesibilidad simbólica muestra que no sólo pueden existir barreras relacionadas con diferencias en los mundos culturales de beneficiarios y profesionales, sino también referidas a construcciones de sentidos individuales, grupales o colectivos que no refieren a diferencias culturales. Así, teniendo en cuenta ambos enfoques y buscando generar una conceptualización más amplia de cómo los sentidos, perspectivas, creencias y expectativas, tanto de los beneficiarios como de los agentes de salud, pueden afectar la accesibilidad, en este trabajo se propone hablar de accesibilidad psicosocio-cultural en lugar de referirse a ella simplemente como "aceptabilidad" de la atención o de accesibilidad "cultural" o "simbólica".

En la Figura 1 se muestra una síntesis de las propuestas conceptuales utilizadas tradicionalmente para pensar la accesibilidad.

\section{La dimensión psico-socio-cultural de la accesibilidad}

Diversos autores han destacado un menor acceso al sistema de salud y/o condiciones de salud más desfavorables en grupos sociales minoritarios o desfavorecidos. Artigas-Lelong \& BennasarVeny 24 han señalado que en España, los inmigrantes que provienen de contextos culturales diferentes y/o desconocen el idioma español encuentran más obstáculos para acceder a los servicios de salud, tienen más dificultad para obtener diagnósticos apropiados y poseen menor adhesión a los tratamientos. También se ha indicado en Estados Unidos que adolescentes y mujeres jóvenes afroamericanas o hispánicas, o simplemente sectores de bajos ingresos, poseen índices mayores de abortos, embarazos no deseados y enfermedades de transmisión sexual 34 Incluso, hay estudios que muestran que estos contrastes entre grupos sociales diferentes se mantienen, aún controlando variables como nivel de ingreso y accesibilidad geográfica 19. En consecuencia, resulta claro que las diferencias culturales pueden tener impactos patentes en el acceso al sistema de salud y a sus beneficios.

La revisión bibliográfica realizada muestra que factores psico-socio-culturales pueden funcionar tanto como barreras o como facilitadores. Por ejemplo, respecto de las prácticas sexuales, en una región rural de India, se observó que varones solteros se sentían avergonzados para acceder a preservativos en sus propios poblados, debido a la existencia de normas sociales que lo consideraban inapropiado 35 . Igualmente, jóvenes no heterosexuales en Estados Unidos reportaron mayor reticencia para acceder a los servicios de salud, debido a temores referidos a la confidencialidad de sus prácticas y a las opiniones negativas que podrían tener los profesionales 36. En relación al fenómeno de la migración, en España se indicó que pacientes provenientes de otras culturas pueden referir síntomas y enfermedades que no son reconocidas en el sistema sanitario local 26. Igualmente, en Estados Unidos se ha señalado que mujeres afroamericanas, latinas y caucásicas comprenden y experimentan 
Propuestas tradicionales para el estudio de la accesibilidad.
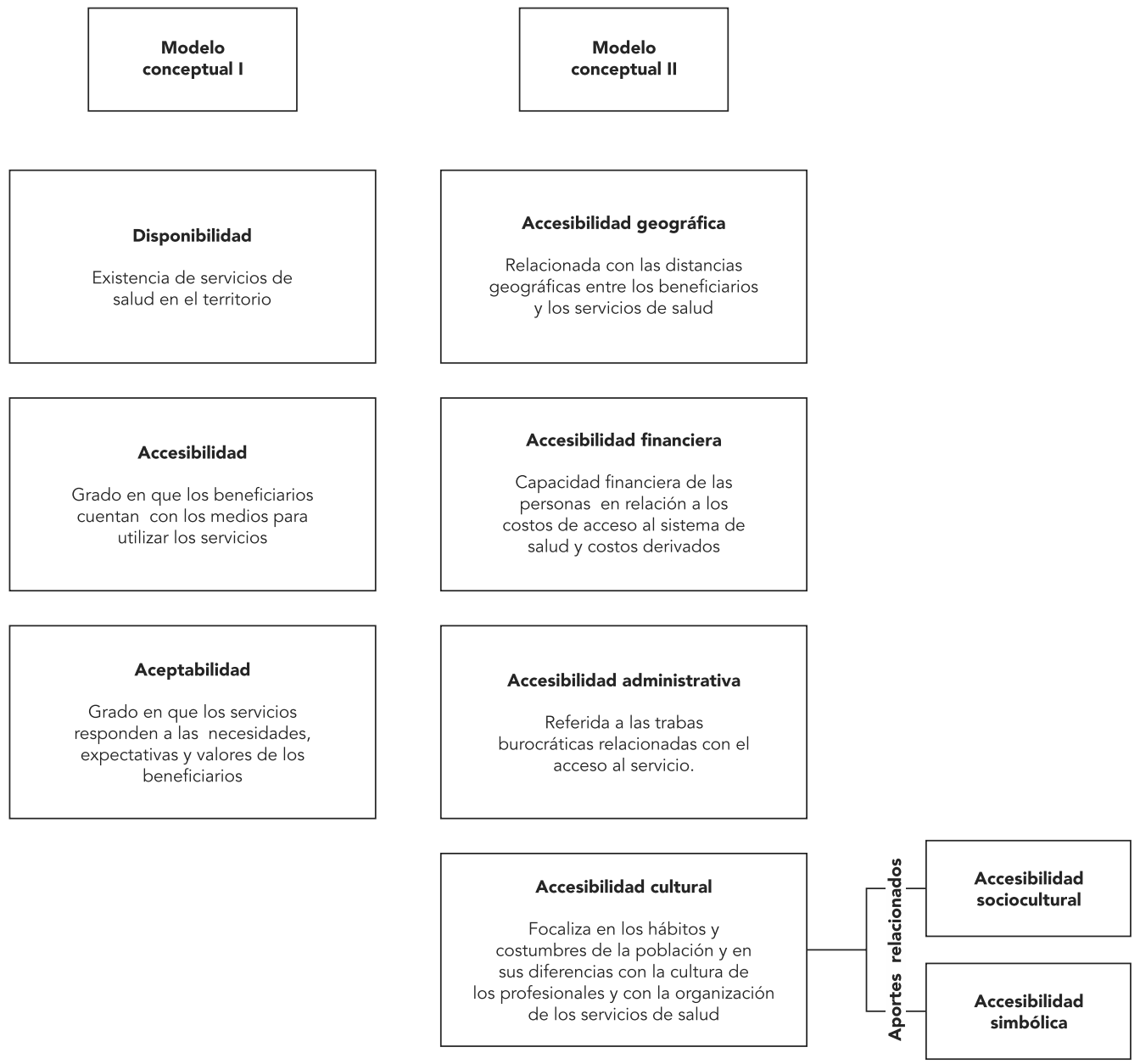

de manera diferente el cáncer de mama, lo que posee impacto en su tratamiento 37 . A su vez, los latinos, también en Estados Unidos, poseen actitudes claramente más fatalistas que otros grupos frente a la diabetes tipo 2, lo que los lleva a menores acciones para afrontar su situación 38 . No obstante, resulta valioso señalar que los factores psico-socio-culturales no siempre funcionan como barreras, sino que también pueden hacerlo como facilitadores, como se observa en un caso en cual se trabajó con refugiados indonesios en Estados Unidos con el apoyo de un pastor cristiano que ofició de intermediario cultural 39 , y en una intervención realizada en el mismo país para detección de cáncer de mama en zonas rurales con el apoyo de iglesias locales 40 .
Los ejemplos mencionados evidencian el interés creciente que ha generado el estudio de las creencias, valores, actitudes y preferencias de los usuarios y su impacto en la atención recibida y en su salud 25. En este sentido, diferentes autores han destacado la importancia de tomar en consideración la confianza de los sujetos en el sistema de atención médica, así como sus creencias relativas a la salud 41 , en particular a las teorías explicativas de la enfermedad ${ }^{9}$, las cuales pueden estar influidas tanto por la etnicidad y la cultura, como por creencias grupales o personales. A su vez, si bien menos extensamente, también se ha mencionado el rol de las creencias y las actitudes de los profesionales, destacándose factores como la dificultad para transmitir información de 
manera comprensible a los pacientes 42 o su escasa sensibilidad para trabajar con diferencias culturales o de género 24,30,38. En resumen, se pone de manifiesto que pacientes y profesionales de la salud pueden formar parte de grupos sociales diferentes, los cuales muchas veces poseen creencias, percepciones, concepciones y expectativas diferentes 43 , lo que puede llevar a conflictos, desacuerdos (explícitos o implícitos), así como a una atención y a un tratamiento insatisfactorio ${ }^{44}$. Y esto, particularmente (pero no exclusivamente), en el caso de que ambos tengan raíces culturales o étnicas diferentes. Así, dado que la accesibilidad es un vínculo construido entre usuarios y servicios de salud, resulta fundamental atender tanto a las representaciones y creencias de los beneficiarios, como a los marcos de sentido construidos por los profesionales y por los servicios de salud 22,23, sin asumir que el problema son las creencias o la cultura de los beneficiarios, sino las dificultades para la articulación entre sus creencias y cultura y las estructuras de sentido en la que se basan los servicios de salud y sus profesionales.

En cierto sentido, los estudios de la percepción de los usuarios respecto a la atención recibida han permitido recuperar la perspectiva de los pacientes en tanto sujetos, y no ya como objetos, cuyas creencias o especificidades culturales son vistas como barreras. Los estudios sobre satisfacción de los usuarios o de calidad desde la perspectiva del usuario se proponen evaluar los servicios para repensar el modo en que éstos se encuentran organizados para mejorar la atención 4,15,21,45. No obstante, dado que la mayor parte de estos estudios han sido realizados desde una perspectiva cuantitativa 27 , generalmente usando encuestas de satisfacción, se hace difícil reconstruir verdaderamente la perspectiva de los beneficiarios, ya que las categorías, ejes o reactivos de evaluación suelen ser construidos desde el punto de vista externo de los especialistas, quedando por fuera la especificidad de la perspectiva del beneficiario 3 , el cual puede contar con herencias culturales o pertenencias sociales que llevan a puntos de vista distantes de los de los evaluadores. Igualmente, existen estudios que muestran que las evaluaciones de satisfacción pueden llevar a resultados contrapuestos, respecto si son realizados desde una metodología cuantitativa o una cualitativa 17 .

Los estudios en el área de alfabetización en salud también contribuyen al estudio de la accesibilidad psico-socio-cultural al sistema de salud. De todas maneras, estas contribuciones se han dado particularmente en el ámbito anglosajón, ya que se observa escaso desarrollo de la temática en el contexto latinoamericano 46,47. En términos generales se define a la alfabetiza- ción en salud como la capacidad de las personas para obtener y procesar información, con el fin de tomar decisiones informadas que les permitan prevenir enfermedades y promover su salud 46,48,49. Diferentes autores argumentan que una limitada alfabetización en salud puede convertirse en una barrera que limite el acceso al sistema de salud 47,50,51. Vista así, si bien la noción de alfabetización en salud constituye un elemento de gran interés para pensar la accesibilidad psico-socio-cultural, también presenta dos limitaciones. Primero, la idea de alfabetización en salud se centra en los conocimientos y capacidades de los usuarios, no en las estrategias de los profesionales o en las estructuras de los sistemas de salud. Encuadrado así el problema, las propuestas de acción se orientan a buscar mejores maneras para educar a los pacientes, para explicarles sus padecimientos y para formular recomendaciones $46,47,49$. Es cierto que diferentes autores han señalado la necesidad de no pensar en términos de responsabilidad de los pacientes, sino de un desafío para el sistema de salud 48,49,52. De cualquier manera, resta el problema de partir de una visión centrada en los profesionales de lo que es (o debería ser) la alfabetización en salud de los pacientes, en el sentido de asumir a priori cuáles son los conocimientos y prácticas correctos que deberían ser seguidas por ellos y que, por tanto, hay que indicarles. De esta manera el diálogo, orientado a la comprensión del punto de vista, las preocupaciones y visiones personales o culturales de la enfermedad, queda desplazado por la "necesidad" de una buena pedagogía para transmitir (transferir) lo que los pacientes deberían saber y hacer. Sin pretender negar el valor intrínseco de la noción de alfabetización en salud, ¿̇no será posible pensar que en muchos casos se la confunda simplemente con diferencias culturales? Ante esto, diferentes aportes de autores han señalado que la alfabetización en salud no puede ser ni identificada con lo que son conocimientos y prácticas 'occidentales', ni evaluada de manera descontextualizada. Como señalan Martensson \& Hensing 49 (p. 5) "es posible que un mismo individuo sea alfabetizado en salud en un contexto o en una sociedad particular, pero no en otro". Por ejemplo, en un estudio realizado en los Estados Unidos 53 pudo observarse que refugiados somalíes que mantenían prácticas tradicionales tenían menos problemas de salud bucal que aquellos con un grado intermedio de aculturación, el cual los había llevado a perder modos de prevención propios de su cultura de origen. Así, surge lo que Chinn 54 denomina "segunda camada” de estudios de alfabetización en salud apoyados en una perspectiva intercultural. 
Los trabajos realizados sobre el vínculo entre pacientes y profesionales de la salud también han abordado la perspectiva de los usuarios, incluyendo sus preferencias y expectativas, así como el impacto tanto de sus creencias como de su cultura en el vínculo con los profesionales y en su salud. Numerosos autores han destacado el impacto que tiene este vínculo en la construcción de diagnósticos apropiados, en la adherencia a los tratamientos, la construcción de confianza interpersonal y, en general, en la efectividad de la atención médica brindada $18,25,55,56,57,58$. Este abordaje, a diferencia de los estudios de satisfacción de los usuarios o encuadrados desde la perspectiva de alfabetización en salud, sí ha permitido focalizar en las representaciones, actitudes, capacidades y conductas de los profesionales de la salud y de todo el equipo médico 24, lo que permite prestar atención a sus respuestas, frente a la diferencia cultural y a las diferencias entre las creencias de profesionales y beneficiarios. De esta manera, la atención no queda focalizada en los "problemas culturales" de los beneficiarios, como responsables del "problema", sino en la interacción de las características psico-socio-culturales de los pacientes y los marcos de sentido, a partir de los cuales se organizan los servicios de salud y sus agentes. Es decir, algo compartido.

Con el fin de enfrentar las barreras derivadas de diferencias en la cultura y/o las creencias o percepciones de pacientes y profesionales, se han planteado múltiples propuestas y se han destacado varios aspectos de la relación. En primer lugar, se ha propuesto avanzar hacia un enfoque intercultural en el cual se comprendan y respeten los sentidos que la salud y la enfermedad tienen para los otros 24 , lo que bien podría colocarse dentro de las propuestas actuales en torno a la implementación de un modelo de atención centrado en el paciente ${ }^{59}$. Dentro de este modelo, se propone el establecimiento de relaciones colaborativas entre profesionales y beneficiarios en las que se evite el establecimiento de vínculos jerárquicos o de poder entre ambos 16,59,60,61, con el fin de favorecer la comunicación y la expresión de las expectativas, temores, percepciones y creencias de los pacientes 60,62 . En esta línea, resulta particularmente inapropiado dejar de atender o incluso rechazar o criticar el punto de vista y las acciones de los pacientes 9,18,38,48, aun cuando estos vayan en contra de las recomendaciones médicas, ya que una propuesta de estas características no hace sino invitar a los pacientes a ocultar sus verdaderas acciones y puntos de vista, lo que limita las posibilidades de los profesionales de intervenir sobre ellas. Asimismo, también se ha mencio- nado la importancia de que los profesionales se expresen con los pacientes de manera clara y comprensible, ya que el excesivo uso de tecnicismos puede llevar a la percepción de trato inadecuado 63. De esta forma, la capacidad de escucha 61 y las habilidades de comunicación de los profesionales 43,57,64, así como su competencia cultural 44,65, quedan ubicadas en un lugar fundamental a la hora de superar las barreras psico-socio-culturales que pueden surgir en el vínculo entre los profesionales y los servicios de salud, y los pacientes.

\section{Hacia un marco conceptual para el abordaje de la accesibilidad psico-socio-cultural}

Con el fin de generar un entramado conceptual que permita abordar de manera específica la accesibilidad psico-socio-cultural, resulta necesario atender a la dimensión relacional a partir de la cual se articula la accesibilidad, focalizando tanto en las diferencias en las representaciones, actitudes y prácticas entre el sistema de salud, sus profesionales y los beneficiarios o pacientes, como en los conflictos que pueden surgir entre ellos. Entendida así, la accesibilidad psico-sociocultural puede ser conceptualizada como una interfaz social. Long 66 (p. 445) define a la interfaz social como "un punto crítico de intersección entre diferentes mundos de vida, campos sociales o niveles de organización social en donde es más probable localizar discontinuidades sociales, basadas en discrepancias en valores, intereses y poder". Este concepto resulta interesante, ya que permite poner el foco en las discontinuidades que se observan, en el contexto de la atención médica, entre los profesionales de la salud (portadores de saberes médicos o científicos) y los pacientes (poseedores de saberes empíricos, experienciales o cotidianos), situación que se traduce en diferentes intereses y prioridades. Siguiendo a Long \& Villarreal 67 es importante señalar que, dadas las diferencias que existen en los marcos de sentido que guían las conductas de profesionales y pacientes en el contexto de la interacción en las interfaces, será posible observar conflictos, acomodamientos e imposiciones entre ellos, en relación a qué es lo que debe hacerse para enfrentar un determinado problema de salud, y a qué debe considerarse como conocimiento verdadero y a qué como prejuicio o como mera "creencia cultural".

En el contexto de la salud, el concepto de interfaz puede ser utilizado para estudiar el modo en que se implementan políticas, iniciativas o acciones en salud que llegan a la población. 
Tradicionalmente, se ha considerado que si los pasos de los procesos de planificación e implementación de acciones eran llevados adelante, podrían alcanzarse los resultados deseados 68 . No obstante, si se asume la existencia de diversos actores sociales implicados, es necesario admitir con ello que los planificadores, las autoridades institucionales, los profesionales de la salud y los pacientes provenientes de distintos entornos socioculturales pueden contar con intereses, objetivos, prioridades y valores propios, y por ende, con una racionalidad distintiva 69,70 , no necesariamente coherente o afín con la del resto de los actores involucrados. Así, el eje del estudio de las interfaces sociales, en el contexto de la accesibilidad, será el análisis de los procesos de negociación y conflicto que se dan, en distintos niveles y entre los diferentes actores, en torno a acciones, propuestas, recomendaciones y conocimientos legítimos, basados en posiciones institucionales o en marcos de sentido diferentes. A la vez, esto deberá complementarse con el seguimiento de las cadenas de traducciones (correcciones, desplazamientos y traslados), que sufren las políticas, los lineamientos institucionales y las recomendaciones médicas a raíz de los intereses distintos y/o contrapuestos de los actores involucrados, específicamente, en el vínculo entre profesionales y pacientes.

Visto desde estas referencias conceptuales, fenómenos tan comunes como el incumplimiento de prescripciones médicas, la combinación de tratamientos recomendados con otro tipo de estrategias (como remedios caseros, por ejemplo) 28, así como el ocultamiento o distorsión de estas acciones en el vínculo con los profesionales, pueden ser pensados como formas de apropiación y relaboración de las recomendaciones e interpretaciones médicas por parte de los pacientes a partir premisas, intereses, prioridades y contextos que no se identifican con los de los profesionales. En consecuencia, el espacio y la dinámica de interacción que se genera en el vínculo adquiere importancia fundamental, ya que será el tipo de interacción que se genere la que permitirá hacer de estas diferencias objeto de reflexión o, por el contrario, invitará a ocultarlas bajo relaciones de poder, generalmente no explicitadas, que limitarán la posibilidad de un diálogo sincero y abierto. Ciertamente, este esquema puede hacerse más complejo analizando diferentes niveles o cadenas de traducción. Por un lado, puede estudiarse cómo las acciones de los profesionales se vinculan con políticas públicas, con lineamientos institucionales o con los recursos materiales disponibles en sus ámbitos de trabajo (como, por ejemplo, el diagnóstico instrumental de distintos tipos), pudiéndose ubicar en este ni- vel espacios de apropiación y traducción, como ocurre cuando unidades de salud territoriales toman las políticas que llegan de niveles decisorios superiores y las aplican según sus preferencias y posibilidades. Por otra parte, también resulta posible estudiar los procesos por medio de los cuales las concepciones, creencias y prioridades de los diferentes actores, especialmente profesionales de la salud y pacientes, son reproducidas y sostenidas en el contexto del vínculo con grupos de pares 71 .

En la Figura 2 puede observarse una síntesis de la propuesta conceptual desarrollada para abordar la accesibilidad psico-socio-cultural de la población al sistema de salud. Finalmente, en la Tabla 1 se comparan los abordajes tradicionales de la accesibilidad cultural con la propuesta conceptual desarrollada en este subtítulo.

\section{Reflexiones finales}

En este trabajo, se reflexionó críticamente sobre la noción de accesibilidad, específicamente, en lo que se refiere a la accesibilidad psico-sociocultural. Se argumentó la falta de soportes conceptuales de numerosos trabajos que abordan el tema y se señaló la limitación de la intuición "espacial" que induce la noción de accesibilidad frecuentemente utilizada, lo que lleva a focalizarse en el acceso de la población beneficiaria al sistema de salud, como un valor en sí mismo, identificándose el acceso al sistema sanitario con la obtención de una atención que genere efectos positivos en la salud de los beneficiarios. Esta falacia "geográfica" o "espacial" de la imagen que tiende a generar la noción de accesibilidad tiene implicaciones particularmente significativas, ya que lleva a considerar como sinónimos el acceso al sistema y los beneficios de salud que éste puede generar en las personas. De esta manera, quedan invisibilizados, a los ojos de planificadores e incluso de expertos, los factores que inciden en los resultados de esta atención, que se relacionan con la interacción entre los beneficiarios y el sistema de salud y sus agentes, así como los marcos culturales, ideológicos y materiales que dan forma a esta interacción.

Con el fin de mostrar la relevancia del nivel psico-socio-cultural de la accesibilidad, se presentaron numerosos ejemplos enmarcados en distintos abordajes. No obstante, se señaló la ausencia de un marco conceptual consolidado para el estudio de la dinámica en su complejidad, lo que dio pie a la presentación de la noción de interfaz social. A partir de ella, se propuso focalizar en las diferencias en los mundos culturales y de sentido, y en las dinámicas de interacción entre 
Figura 2

Propuesta conceptual para el abordaje de la accesibilidad psico-socio-cultural.

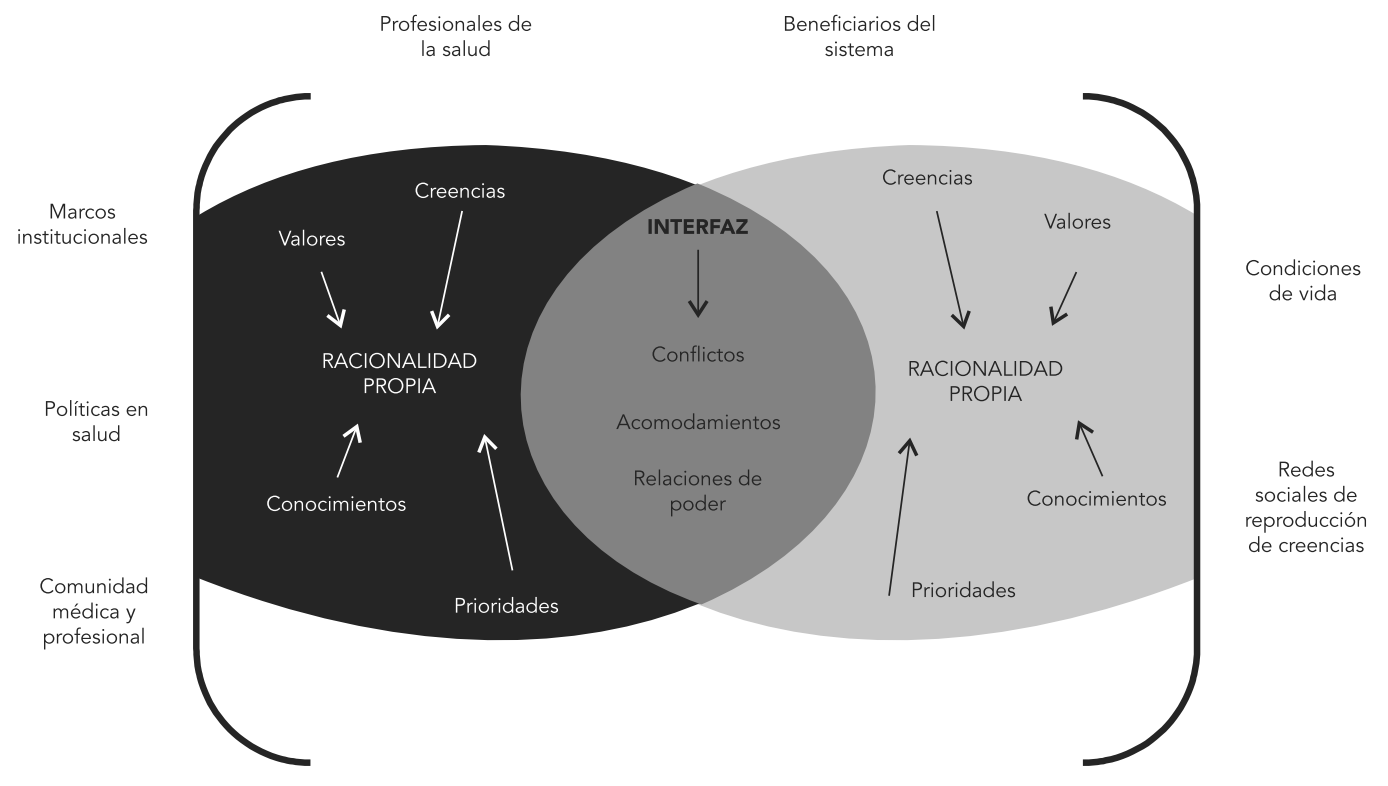

Tabla 1

Comparación entre modelos conceptuales para abordar la accesibilidad cultural.

\section{Ejes de comparación}

1. Soporte conceptual

2. Concepción de cultura de profesionales y beneficiarios en las barreras culturales

\section{Dinamismo e} historicidad

5. Modo de estudio/ evaluación

6. Grado de uso a nivel científico

\section{Abordaje tradicional de la accesibilidad cultural}

Débil, construido a partir de definiciones y construcción de tipologías.

Sesgo etnocéntrico al focalizarse en la cultura de 'los otros' sin dar valor particular al carácter culturalmente determinado de los enfoques médicos y de las instituciones en las que ellos trabajan.

Limita el análisis de barreras de acceso a la dimensión cultural.

El foco es puesto en el beneficiario. Su cultura tiende a observarse como una barrera. El profesional asume la responsabilidad de facilitar la comunicación para reducir problemas potenciales.

Tendencia a adoptar una mirada estática de las barreras de nivel cultural. Da limitada atención a la interacción profesional-beneficiario

Mayormente cuantitativo, apoyado en la realización de encuestas/cuestionarios.

Alto reconocimiento, amplia presencia en la bibliografía especializada.

\section{Accesibilidad psico-socio-cultural como interfaz social}

Noción de interfaz social. Apoyada en la tradición de la sociología orientada al actor 66

Reconocimiento de la diversidad cultural. Los marcos culturales y subjetivos de los beneficiarios son reconocidos al igual que aquellos que portan los profesionales y sus instituciones. Incluye en su conceptualización tanto diferencias culturales, como otras derivadas de creencias, o valores tanto individuales como grupales o colectivos, sin necesidad de que refieran a factores estrictamente culturales.

La accesibilidad a nivel cultural es pensada en términos de falta de acople entre ambos elementos: las concepciones y situación de los beneficiarios y las de los profesionales,

enmarcados en el contexto del sistema de salud.

Concepción dinámica de los procesos por medio de los cuales las propuestas son transformadas por los distintos actores.

Preferentemente cualitativo, centrado en la reconstrucción y comprensión de la visión de los actores, aunque con posibilidad de cuantificación.

Propuesta alternativa. 
beneficiarios y profesionales de la salud que se generan a partir de ellas, siempre teniendo en cuenta las estructuras institucionales en las cuales esta interacción usualmente tiene lugar. En este contexto, se procuró focalizar en el análisis de los vínculos, muchas veces conflictivos, que se dan entre los beneficiarios, los profesionales y las instituciones de salud, y los procesos por los cuales pacientes y profesionales se apropian, reinterpretan o rechazan los pareceres y las recomendaciones del otro actor.

A continuación se indican las implicaciones que se desprenden de este trabajo.

Implicaciones para gestores de salud y autoridades institucionales

Es necesario tomar conciencia que el acceso de los beneficiarios al espacio físico de las instituciones de salud no es suficiente. Por esto, hay que atender seriamente a la dinámica que media entre el acceso al sistema y la obtención de beneficios en términos de salud por parte de los beneficiarios, lo que lleva a centrarse en las barreras psico-socio-culturales que pueden aparecer en ese proceso. Esto exige formar profesionales y agentes de salud con capacidad para trabajar con personas con una cultura, una concepción de salud y enfermedad y un contexto de vida que no se identifica con el suyo propio. A la vez, se necesita que la estructuración misma del sistema de salud, de los programas de asistencia, de las condiciones materiales en las que se brinda esa atención y de las exigencias que se imponen a los profesionales y agentes de salud, sean consistentes con -o sensibles a- la racionalidad, los valores y las creencias de la población beneficiaria. Si la estructura misma del sistema y de la atención no es permeable a los marcos de sentido con los que se manejan los beneficiarios, resultará cuanto menos ingenuo asignar responsabilidades a los usuarios, cuando estos no respondan como se espera a algunas de las propuestas que se les realizan. Esto implica estructurar la atención en salud, no sólo a partir de una lógica médica y una administrativa, sino también teniendo en cuenta los marcos de sentido (culturales y de otro tipo) que traigan los beneficiarios.

\section{Implicaciones para profesionales de la salud}

Existe una tendencia de los profesionales de la salud a pensar su estrategia de atención a partir de la lógica médica aprendida en su formación. Esto lleva a entender fenómenos como la falta de adherencia a tratamientos o la falta de seguimiento de recomendaciones, poniendo gran parte de la responsabilidad en los beneficiarios. Esto refleja cierta limitación para reconocer que los otros, los pacientes, los que solicitan atención, tienen formas de pensar e interpretar, creencias y contextos de vida propios con los cuales es necesario que dialoguen las recomendaciones y tratamientos propuestos. Difícilmente, una embarazada que tiene que acarrear agua en baldes para dar de tomar a sus hijos deje de hacer ese esfuerzo, pese a que el médico le diga que no se esfuerce porque está embarazada. De esto se sigue, primero, que los profesionales de la salud deben reflexionar críticamente sobre sus propias prácticas y acciones (en lugar de focalizar en los otros), evaluando de qué manera están contribuyendo con ellas para llegar a resultados o situaciones indeseadas. Segundo, que es necesario ser sensibles a las diferencias culturales, de creencias y de contextos de vida que marcan a los pacientes. Se necesita aquí capacidad para entrar en diálogo con los otros, escuchar su punto de vista, no sólo chequear si entienden lo que se les recomienda, sino dialogar para saber qué piensan de ello y cuáles son las posibilidades reales de hacerlo.

\section{Implicaciones para la investigación}

Las investigaciones sobre accesibilidad cultural tienden a utilizar formatos cuantitativos que la reducen a encuestas de satisfacción respecto de la atención recibida. Del esquema conceptual propuesto se deriva un conjunto de líneas de investigación mucho más amplio y rico que incluye la indagación de cómo los marcos institucionales y los programas de salud constriñen y dan forma a las prácticas de atención, cómo profesionales y agentes de salud se apropian y transforman esas acciones y políticas que deben llevar adelante, cómo se da el vínculo entre profesionales y pacientes, y cómo estos últimos toman y reconfiguran a partir de sus propias creencias y contexto de vida las recomendaciones de profesionales y agentes de salud. En este sentido, las visiones que profesionales y pacientes tienen uno del otro resulta un elemento fundamental, en tanto estas representaciones contribuyen a dar forma a la dinámica del vínculo entre ellos y al seguimiento de las recomendaciones médicas. Si bien lo planteado apunta a la realización de estudios cualitativos orientados a comprender las concepciones, tanto de beneficiarios como de profesionales y agentes de salud, existen múltiples espacios para la investigación cuantitativa, como ser la cuantificación de las creencias que profesionales y potenciales beneficiarios tienen uno del otro y que tienen los últimos sobre la enfermedad y la salud, la evaluación del impacto de distinto tipo de dinámicas de interacción entre profesionales y pacien- 
tes e, incluso, la evaluación psicométrica de la capacidad de los profesionales para incorporar en sus intervenciones los requerimientos de la cultura, creencias y contexto de vida de los beneficiarios. En consecuencia, se observa que se abren diferentes líneas de investigación, tanto cualitativas, como cuantitativas.

\section{Resumo}

A acessibilidade da população ao sistema de saúde é uma das principais preocupações das políticas públicas. No entanto, são poucos os artigos que aprofundam conceitualmente tal noção. A maioria deles apresenta diferença entre disponibilidade, acessibilidade e aceitabilidade, ou entre acessibilidade geográfica, financeira, administrativa e cultural. Neste artigo, discute-se a noção de acessibilidade, que é definida como um processo de articulação complexo entre oferta e demanda em saúde. Ao mesmo tempo, estuda-se em profundidade a noção de acessibilidade cultural, a qual é repensada como uma interface social, ou seja, como um espaço em conflito no qual se articulam diferentes mundos de significado, nesse caso, os dos profissionais e dos pacientes. Isto permite abordar os complexos processos de apropriação, tradução e reconfiguração do conhecimento e recomendações que ocorrem nesse relacionamento.

Acesso aos Serviços de Saúde; Fatores Culturais; Fenômenos e Processos Psicológicos; Barreiras de Comunicação
Finalmente, hay que recordar que el presente trabajo se apoya en una propuesta conceptual abierta, inacabada. Para clarificar su pertinencia y utilidad resulta necesario continuar con la discusión conceptual, a la vez que avanzar con las líneas de investigación propuestas para evaluar su utilidad práctica.

\section{Colaboradores}

F. Landini participó en la reflexión conceptual que dio origen al manuscrito, escritura de la mayor parte del manuscrito y aprobación del manuscrito final. V. G. Cowes participó en la reflexión conceptual que dio origen al manuscrito, escritura y revisión crítica del manuscrito y aprobación del manuscrito final. E. D’Amore participó en la reflexión conceptual que dio origen al manuscrito, revisión crítica del manuscrito y aprobación del manuscrito final.

\section{Agradecimientos}

El trabajo de revisión bibliográfica y de reflexión crítica en el que se apoya este artículo fue financiado por una investigación multicéntrica, aprobada por la Comisión Nacional Salud Investiga del Ministerio de Salud de la Nación (Argentina) en el contexto de la convocatoria 2012 de las becas de investigación "Carrillo Oñativia" y por el proyecto de investigación propio de la Universidad de la Cuenca del Plata Accesibilidad Psico-SocioCultural al Sistema de Salud en Pequeños Productores Agropecuarios de Corrientes y Misiones (2011-2013). 


\section{Referencias}

1. Organización Panamericana de la Salud. Declaración de Alma Ata. http://www.paho.org/spanish/ dd/pin/alma-ata_declaracion.htm (accedido el 14/Feb/2013).

2. Organización Panamericana de la Salud. La renovación de la atención primaria de salud en las Américas. http://www.paho.org/Spanish/AD/ THS/OS/APS_spa.pdf (accedido el 14/Feb/2013).

3. Vargas-Lorenzo I, Vázquez-Navarrete ML, Mogollón-Pérez AS. Acceso a la atención en salud en Colombia. Rev Salud Pública 2010; 12:701-12.

4. Bernal-Delgado E, Peiró S, Sotoca R. Prioridades de investigación en servicios sanitarios en el sistema nacional de salud. Una aproximación por consenso de expertos. Gac Sanit 2006; 20:287-94.

5. Arakawa T, Arcêncio R, Scatolin B, Scatena L, Ruffino-Netto A, Scatena Villa T. Accessibility to tuberculosis treatment: assessment of health service performance. Rev Latinoam Enferm 2011; 19: 994-1002.

6. Lacle Murray A. ¿Existe equidad de acceso a los servicios de salud para el adulto mayor según zona de residencia (urbano/rural)? Rev Costarric Salud Pública 1999; 15:57-63.

7. Arredondo A, Nájera P. Equity and accessibility in health? Out-of-pocket expenditures on health care in middle income countries: evidence from Mexico. Cad Saúde Pública 2008; 24:2819-26.

8. Gegúndez-Fernández JA. Modelo de gestión asistencial basado en el principio de accesibilidad total. Arch Soc Esp Oftalmol 2008; 83:147-50.

9. Henderson S, Kendall E. Culturally and linguistically diverse peoples' knowledge of accessibility and utilisation of health services: exploring the need for improvement in health service delivery. Aust J Prim Health 2011; 17:195-201.

10. Moreno GA, Monsalve JC, Tabima D, Escobar JI. Apreciaciones de la población en condición de desplazamiento forzado sobre los servicios de salud en algunos municipios de Colombia. Rev Fac Nac Salud Pública 2009; 27:131-41.

11. Tirado Otálvaro AF, Correa Arango ME. Accesibilidad de la población habitante de calle a los programas de promoción y prevención establecidos por la Resolución 412 de 2000. Investig Andin 2009; 18:23-35.

12. Solitario R, Comes Y, Garbus P, Mauro M, Stolkiner A. Accesibilidad al sistema de salud de una población de adultos mayores: una experiencia participativa en el proceso de investigación en sistemas y servicios de salud. Revista Científica de la AMBB 2006; 16:97-102.

13. Comes Y, Stolkiner A. Si pudiera pagaría: estudio sobre la accesibilidad simbólica de las mujeres usuarias pobres del AMBA a los servicios asistenciales estatales. Anu Investig - Fac Psicol, Univ B Aires 2004; 12:137-43.

14. Travassos C, Martins M. Uma revisão sobre os conceitos de acesso e utilização de serviços de saúde. Cad Saúde Pública 2004; 20 Suppl 2:S190-8.
15. Ramírez-Sánchez TJ, Nájera-Aguilar P, NigendaLópez G. Percepción de la calidad de la atención de los servicios de salud en México: perspectiva de los usuarios. Salud Pública Méx 1998; 40:3-12.

16. Pinho LB, Hernández AMB, Kantorski LP. Discurso sobre o acolhimiento e a acessibilidade nos servicos comunitarios de saúde mental. Cogitare Enferm 2009; 14:612-9.

17. Gaioso VP, Mishima SM. Satisfacción del usuario en la perspectiva de la aceptabilidad en el escenario de la salud de la familia. Abordaje cuali-cuantitativo. Texto \& Contexto Enferm 2007; 16:617-25.

18. Clementino FS, Miranda FAN. Acessibilidade: identificando barreiras na descentralização do controle da tuberculose nas unidades de saúde da família. Rev Enferm UERJ 2010; 18:584-90.

19. Shah BR, Gunraj N, Hux JE. Markers of access to and quality of primary care for aboriginal people in Ontario, Canada. Am J Public Health 2003; 93:798-802.

20. Martins S, Pereira F, Matumoto S, Fortuna C, Bistafa M, Campos A, et al. Assistance in family health from the perspective of users. Rev Latinoam Enferm 2010; 18:436-43.

21. Mishima S, Pereira F, Matumoto S, Fortuna C, Pereira $\mathrm{M}$, Campos $\mathrm{A}$, et al. La asistencia en la salud de la familia bajo la perspectiva de los usuarios. Rev Latinoam Enferm 2010; 18:151-9.

22. Comes Y, Solitario R, Garbus P, Mauro M, Czerniecki S, Vázquez A, et al. El concepto de accesibilidad: perspectiva relacional entre población y servicios. Anu Investig - Fac Psicol, Univ B Aires 2007; 14: 201-9.

23. Solitario R, Garbus P, Stolkiner A Derechos, ciudadanía y articulación en salud: su relación con la accesibilidad simbólica a los servicios. Anu Investig - Fac Psicol, Univ B Aires 2008; 15:263-9.

24. Artigas-Lelong B, Bennasar-Veny M. La salud en el siglo XXI: el reto de los cuidados multiculturales. Revista Index de Enfermería 2009; 18:42-6.

25. Betancourt JR, Green AR, Carrillo JE, AnanehFirempong O. Defining cultural competence: a practical framework for addressing racial/ethnic disparities in health and health care. Public Health Rep 2003; 118:293-302.

26. Girondi JBR, Santos SMA. Deficiência física em idosos e acessibilidade na atenção básica em saúde: revisão integrativa da literatura. Rev Gaúch Enferm 2011; 32:378-84.

27. Delgado-Gallego ME, Vázquez-Navarrete ML, Moraes-Vanderlei L. Calidad en los servicios de salud desde los marcos de sentido de diferentes actores sociales en Colombia y Brasil. Rev Salud Pública 2010; 12:533-45.

28. Herrera Medina N, Gutierrez-Malaver ME, Ballesteros-Cabrera M, Izzedin-Bouquet R, Gómez-Sotelo AP, Sánchez-Martínez LM. Representaciones sociales de la relación médico-paciente en médicos y pacientes en Bogotá, Colombia. Rev Salud Pública 2010; $12: 343-55$. 
29. Pitchforth E, Teijlingen E, Graham W, DixonWoods M, Chowdhury M. Getting women to hospital is not enough: a qualitative study of access to emergency obstetric care in Bangladesh. Qual Saf Health Care 2006; 15:214-9.

30. Baum FE, Bégin M, Houweling TAJ, Taylor S. Changes not for the fainthearted: reorienting health care systems toward health equity through action on the social determinants of health. Am J Public Health 2009; 99:1967-74.

31. Wallace S, Enriquez-Haass V. Disponibilidad, accesibilidad y aceptabilidad en el sistema de atención médica en vías de cambio para los adultos mayores en los Estados Unidos. Rev Panam Salud Pública $2001 ; 10: 18-28$

32. Wallace S, Gutiérrez V. Equity of access to health care for older adults in four major Latin American cities. Rev Panam Salud Pública 2005; 17:394-409.

33. Barbastefano PS, Girianelli VR, Vargens OM. O acesso á assisténcia ao parto para parturientes adolescentes nas maternidades da Rede SUSA. Rev Gaúch Enferm 2010; 31:708-14.

34. Hall K, Moreau C, Trussell J. Determinants of and disparities in reproductive health service use among adolescent and young adult women in the United States, 2002-2008. Am J Public Health 2012; 102:359-67.

35. Char A, Saavala M, Kulmala T. Assessing young unmarried men's access to reproductive health information and services in rural India. BMC Public Health 2011; 11:476.

36. Williams KA, Chap MV. Comparing health and mental health needs, service use, and barriers to services among sexual minority youths and their peers. Health Soc Work. 2011; 36:197-206.

37. Amjadi-Begvand N, Gazarian M, Howland J, Trimis J, Yong F. The ethnicity of breast cancer: cultural discrepancies in diagnosis and treatment decision. Global Studies Journal 2011; 3:77-84.

38. Caballero A, Tenzer P. Building cultural competency for improved diabetes care: Latino Americans and diabetes. J Fam Pract 2007; 56 Suppl 9:S7-13.

39. Mancuso L. Overcoming health literacy barriers; a model for action. J Cult Divers 2011; 18:60-5.

40. Ka'opua LS, Park SH, Ward ME, Braun K. Testing the feasibility of a culturally tailored breast cancer screening intervention with native Hawaiian women in rural churches. Health Soc Work 2011; 36:55-65.

41. Arredondo A. Factores asociados a la búsqueda y uso de servicios de salud: del modelo psicosocial al socioeconómico. Salud Ment 2010; 33:397-408.

42. Caprara A, Lins A, Franco SA. Relação paciente-médico: para uma humanização da prática médica. Cad Saúde Pública 1999; 15:647-54.

43. de Dios-Lorente JA, Jiménez-Arias ME. La comunicación en salud desde las perspectivas ética, asistencial, docente y gerencial. Medisan 2009; 13(1). http://bvs.sld.cu/revistas/san/vol13_1_09/ san10109.htm.
44. Manassis K. The effects of cultural differences on the physician-patient relationship. Can Fam Physician 1986; 32:383-9.

45. Eulmesekian P, Peuchot V, Pérez A. Satisfacción de los padres de los pacientes en una unidad de cuidados intensivos pediátricos. Arch. Argent Pediatr 2012; 110:113-9.

46. Bonal-Ruiz R, Marzán-Delis M, Castillo-Frómeta M, Rubán-Alfaro M. Alfabetización en salud en medicina general integral. Perspectivas en Santiago de Cuba. Medisan 2013; 13:126-40.

47. Konfino J, Mejía R, Majdalani M, Pérez-Stable E. Alfabetización en salud en pacientes que asisten a un hospital universitario. Medicina (B. Aires) 2009; 69:631-4.

48. Villaire M, Mayer G. Health literacy: the low-hanging fruit in health care reform. J Health Care Finance 2009; 36:55-9.

49. Paasche-Orlow M. Caring for patients with limited health literacy. A 76-year-old man with multiple medical problems. JAMA 2011; 306:1122-9.

50. Shaw S, Huebner C, Armin J, Orzech K, Vivian J. The role of culture in health literacy and chronic disease screening and management. J Immigr Minor Health 2009; 11:460-7.

51. Carrillo J, Carrillo V, Pérez H, Salas-López D, Natale-Pereira A, Byron A. Defining and targeting health care access barriers. J Health Care Poor Underserved 2011; 22:562-75.

52. Fitzgerald-Bone P, Russo-France K, Aikin K. On break-up clichés guiding health literacy's future. J Consum Aff 2009; 43:185-98.

53. Geltman P, Hunter-Adams J, Cochran J, Doros G, Rybin D, Henshaw M, et al. The impact of functional health literacy and acculturation on the oral health status of somali refugees living in Massachusetts. Am J Public Health 2013; 103:1516-23.

54. Chinn D. Critical health literacy: a review and critical analysis. Soc Sci Med 2011; 73:60-7.

55. Bendapudi N, Berry L, Frey K, Turner-Parish J, Rayburn W. Patients' perspectives on ideal physician behaviors. Mayo Clin Proc 2006; 81:338-44.

56. Hausman A. Modeling the patient-physician service encounter: improving patient outcomes. Journal of the Academy of Marketing Science 2004; 32:403-17.

57. Shipman B. The role of communication in the patient-physician relationship. J Leg Med 2010; 31:433-42.

58. Thom D. Physician behaviors that predict patient trust. J Fam Pract 2001; 50:323-8.

59. von Friederichs-Fitzwater MM, Gilgun J. Relational control in physician-patient encounters. Health Commun 2001; 13:75-87.

60. De la Rosa Legón M, Vega González N, Brito Gómez L. El paradigma médico social y la competencia comunicativa del profesional de ciencias médicas. Rev Habanera Cienc Méd 2010; 9:433-40. 
61. González Menéndez R. La relación médico-paciente y su significado en la formación profesional. Educ Med Super 2003; 17(4). http://bvs.sld. cu/revistas/ems/vol17_4_03/ems02403.htm.

62. Manzoni GC, Torelli P. The patient-physician relationship in the approach to therapeutic management. Neurol Sci 2007; 28:130-3.

63. Herrera-Kiengelher L, Villamil-Álvarez M, Pelcastre-Villafuerte B, Cano-Valle F, López-Cervantes M. Relación del personal de salud con los pacientes en la Ciudad de México. Rev Saúde Pública 2009; 43:589-94.

64. Shukla AK, Yadav SV, Kastury N. Doctor-patient communication: an important but often ignored aspect in clinical medicine. Journal of the Indian Academy of Clinical Medicine 2010; 11:208-11.

65. Paez K, Allen J, Beach M, Carson K, Cooper L. Physician cultural competence and patient ratings of the patient-physician relationship. J Gen Intern Med 2009; 24:495-8.

66. Long N. Sociología del desarrollo: una perspectiva centrada en el actor. México DF: Centro de Investigaciones y Estudios Superiores en Antropología Social; 2007.
67. Long N, Villarreal M. Exploring development interfaces: from knowledge transfer to transformation of meaning. In: Schuurman F, editor. Beyond the impasse: new directions in development theory. London: Zed Press; 1993. p. 140-68.

68. van Woerkum C, Aarts N, van Herzele A. Changed planning for planned and unplanned change. Planning Theory 2011; 10:144-60.

69. Lapalma A. El escenario de la intervención comunitaria. Revista de Psicología de la Universidad de Chile 2001; 10:61-70.

70. Landini F. Racionalidad económica campesina. Mundo Agrario 2011; 12(23). http://www.mundo agrario.unlp.edu.ar/article/view/v12n23a14/195.

71. Gergen K. Realidades y relaciones. Aproximaciones a la construcción social. Barcelona: Paidós; 1996.

Recibido el 16/Feb/2013

Versión final presentada el 20/Sep/2013

Aprobado el 19/Nov/2013 